\title{
Comparaison de quelques mesures en nature et surr modèles réduits hydrauliques
}

\author{
par R. Bomnefille el A. Salomone \\ Laboratoire National d'Hydraulique (Chatou)
}

La comparaison des propagations d'intumescences dans l'estuaire de la Rance sur modele et en nature est satisfaisante. Les mesures de courant nature et modele de l'usine sont difficilement comparables parce que effectuées dans des conditions différentes. La comparaison des remous de l'usine modèle et nature est décevante.

\section{Introduction}

Pendant plus de dix ans, la Région d'Equipement Marémotrice et la Direction des Etudes et Recherches ont œuvré conjointement à la préparation de l'aménagement de l'usine marémotrice de la Rance, avec le double souci de parfaire l'ouvrage tout en assurant, aux usagers de l'estuaire, des conditions au moins aussi confortables qu'auparavant. Il s'agissait donc de prévoir la répercussion de l'ouvrage et de son fonctionnement sur l'environnement, et définir les moyens nécessaires pour se prémunir contre les inconvénients qui pourraient en résulter.

Dans ce but, le Laboratoire National d'Hydraulique a conçu un modèle à fonds fixes à l'échelle du $1 / 150$ représentant l'estuaire; il l'a exploité pendant dix années, jusqu'à la mise en service de l'usine. Ce travail s'est concrétisé par l'édition de quelque quatre-vingts rapports et communications, dont un grand nombre traitent de l'exploitation de l'usine et, par conséquent, de son action dans le futur.

Ces documents constituent un recueil prévisionnel des phénomènes hydrauliques qui, actuellement, régissent l'estuaire soustrait de son rythme naturel. Il importe, après six années d'exploitation, d'en juger et critiquer la perspicacité; plus précisément, les travaux du Laboratoire, en matière de prédictions, se sont-ils révélés exacts, satisfaisants, suffi- sants? Notre propos est donc de juger du «satisfecit» recueilli auprès de l'exploitant d'une part, et des riverains et usagers d'autre part, respectivement préoccupés, l'un de sa mission de dispensateur d'énergie, les autres des commodités de navigation, deux objectifs a priori contradictoires et difficilement conciliables.

\section{Rappel des études relatives à l'exploitation de l'usine}

Exploiter l'usine, c'est adapter aux besoins de la consommation ses fonctionnements, en les enchaînant rationnellement en fonction de la marée. Ces fonctionnements entraînent une modification du régime hydraulique de l'estuaire dans la retenue et aux abords immédiats du barrage. L'usine, en marche normale, retarde la marée dans le bássin de trois heures de marée environ; des débits importants transitent alors à bas niveaux, engendrant des vitesses inhabituelles. Les variations de fonctionnement de l'usine peuvent provoquer des oscillations de courtes périodes, inexistantes en régime naturel de marée.

Ces phénomènes ont comme conséquences: d'une part, une augmentation de l'intensité des courants à certaines heures de la marée et, dans une certaine mesure, une modification de leur direction; d'autre part, une accentuation de la pente de la surface libre de l'estuaire et de la mer au voisinage de l'usine.

Les études en modèle mettant en évidence ces phénomènes concernent :

- les conditions de navigation dans la Rance pendant l'exploitation de l'usine; 
$z(m)$

$n(m)$

a $\left(10^{3} \mathrm{~m}^{3 / s}\right)$

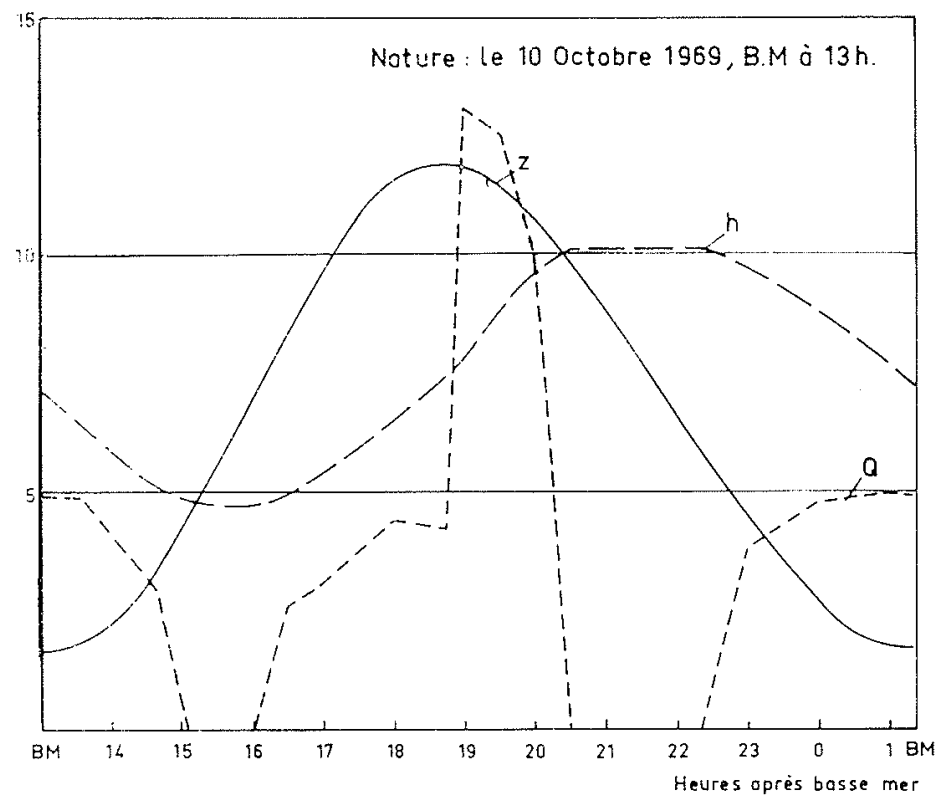

$z(m)$

$h(m)$

$a\left(10^{3} \mathrm{~m}^{3} / \mathrm{s}\right)$

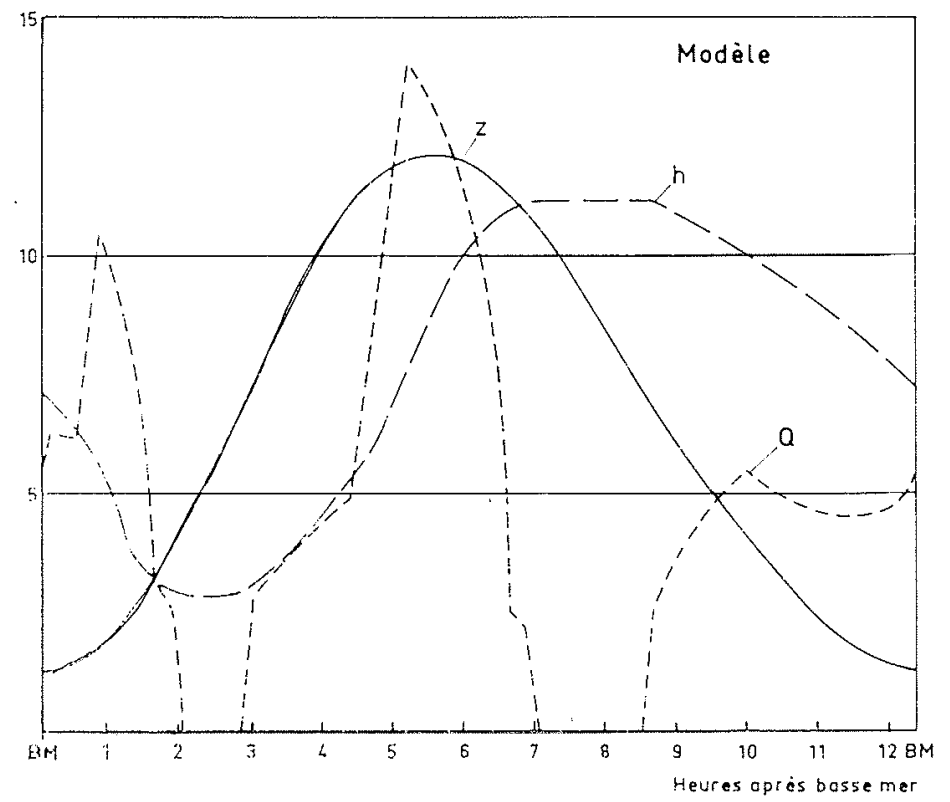

Fis.

$1 /$ $z(m)$
$h(m)$
$a\left(10^{3} m^{3} / 5\right)$

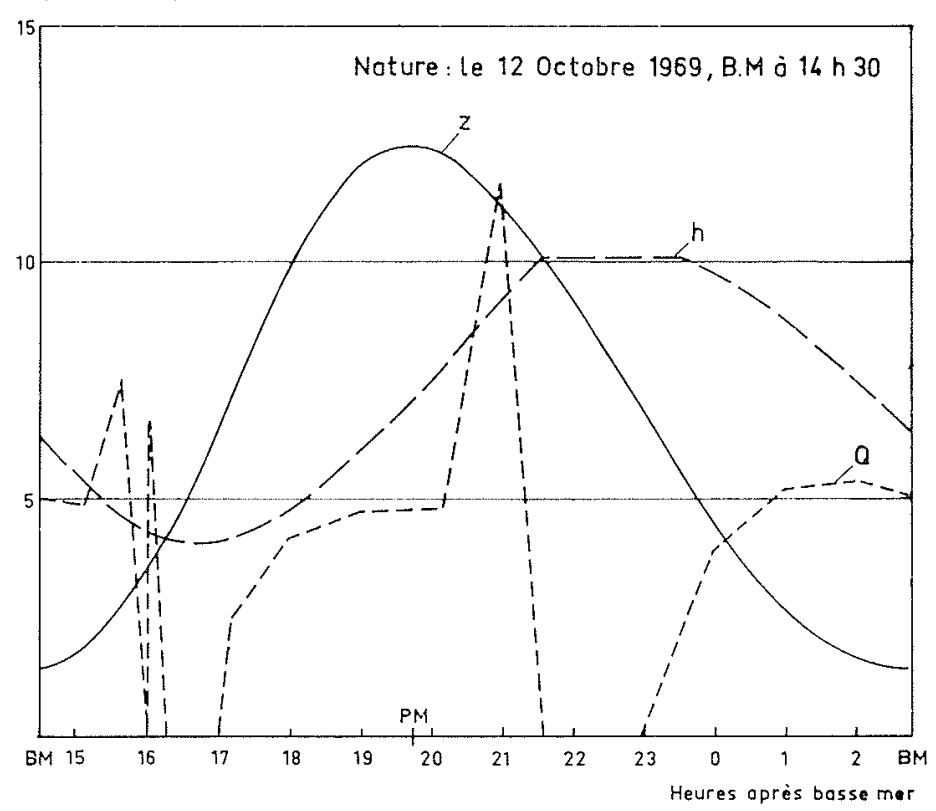

LES CYCLES DE FONCTIONNEMENT MODELE ET NATURE COMPARES
$z$ : cote de la mer
$h$ : cote du bassin
Q : débit traversant les ouvrages 


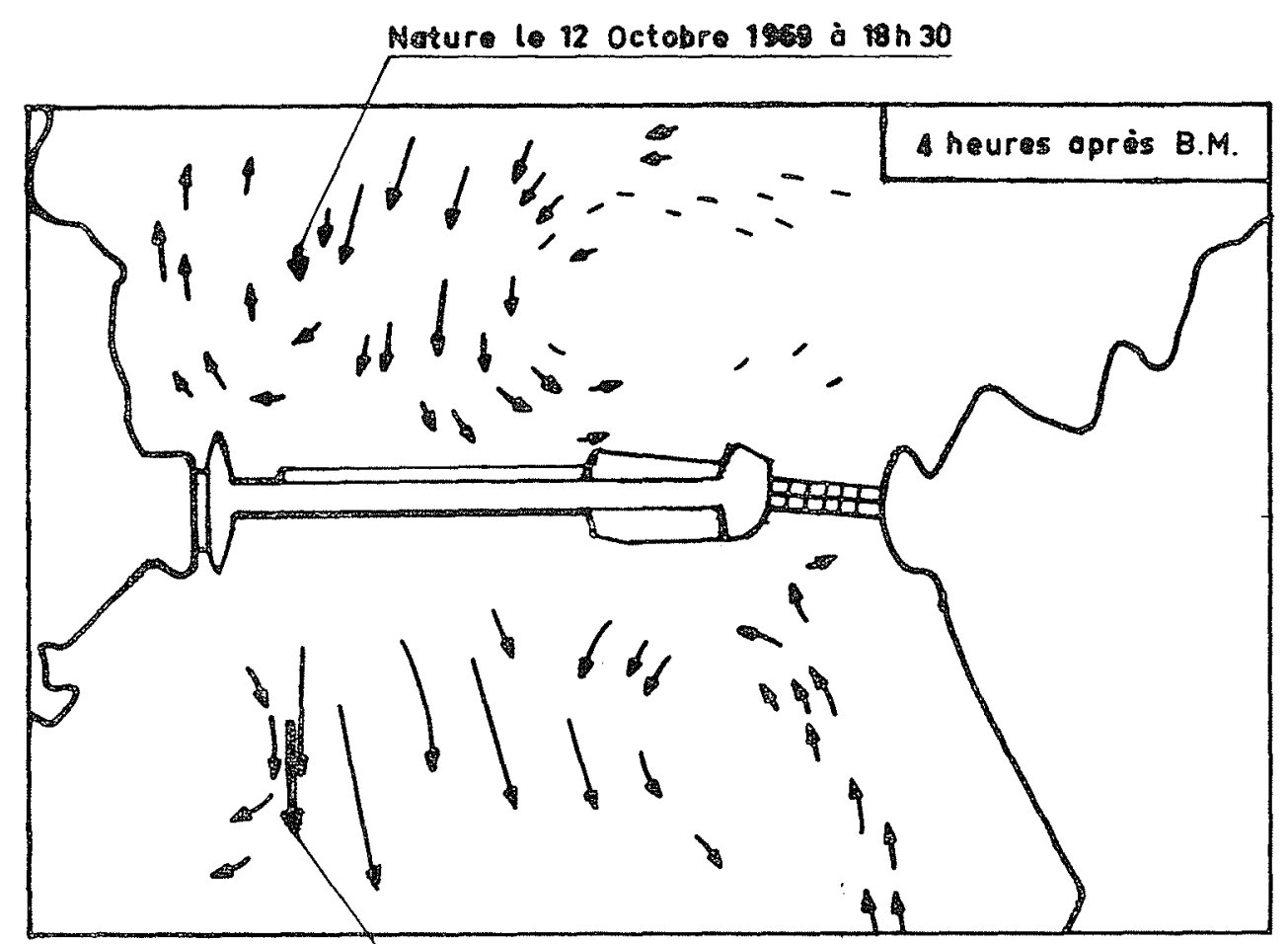

Hoture le 10 Octobre 196 à $19 \mathrm{~h}$

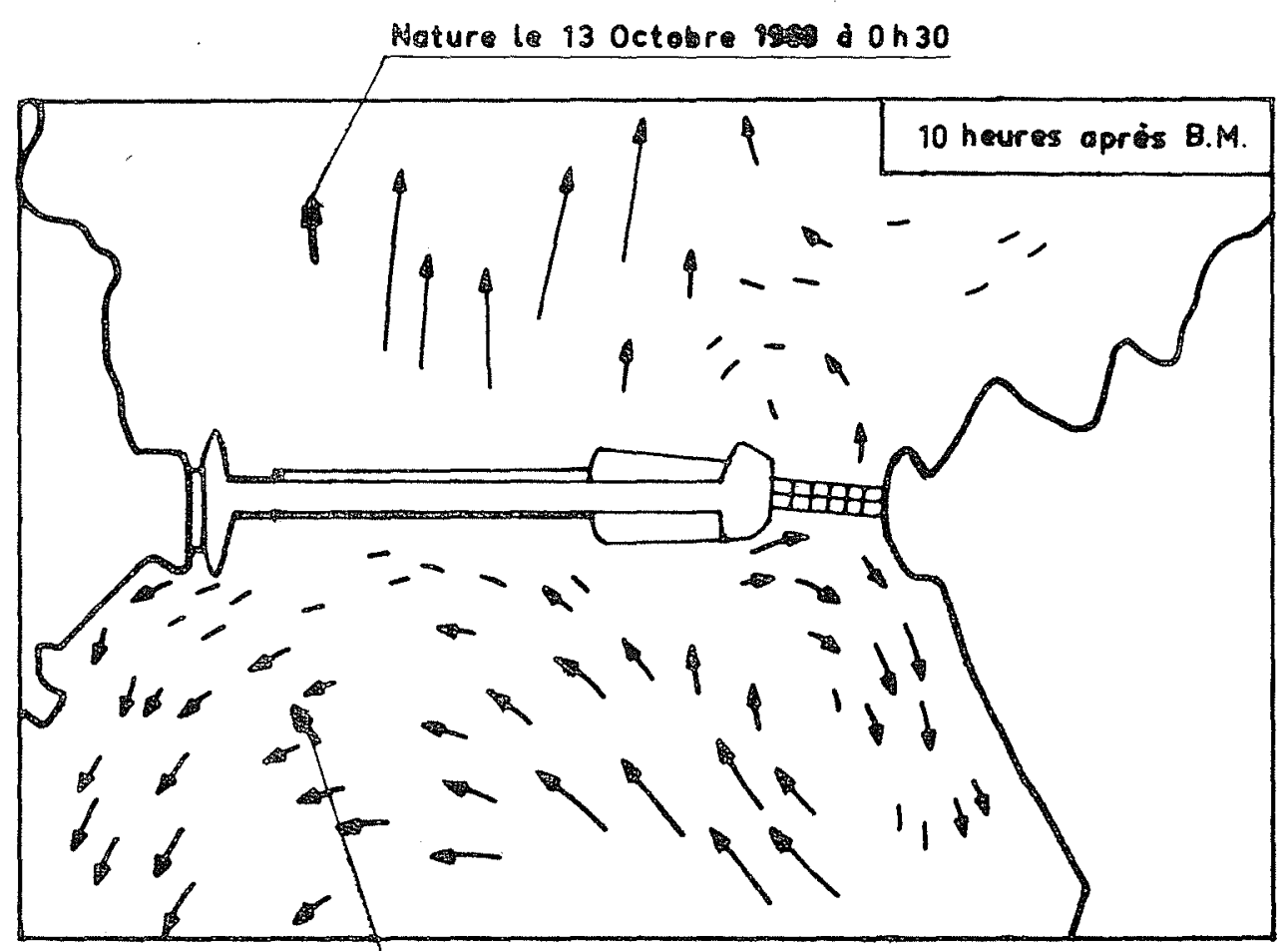

Harure le 10 Oerobro 1969 d $23 n$

2/ Comparaison du champ de courant nature et modèle. 

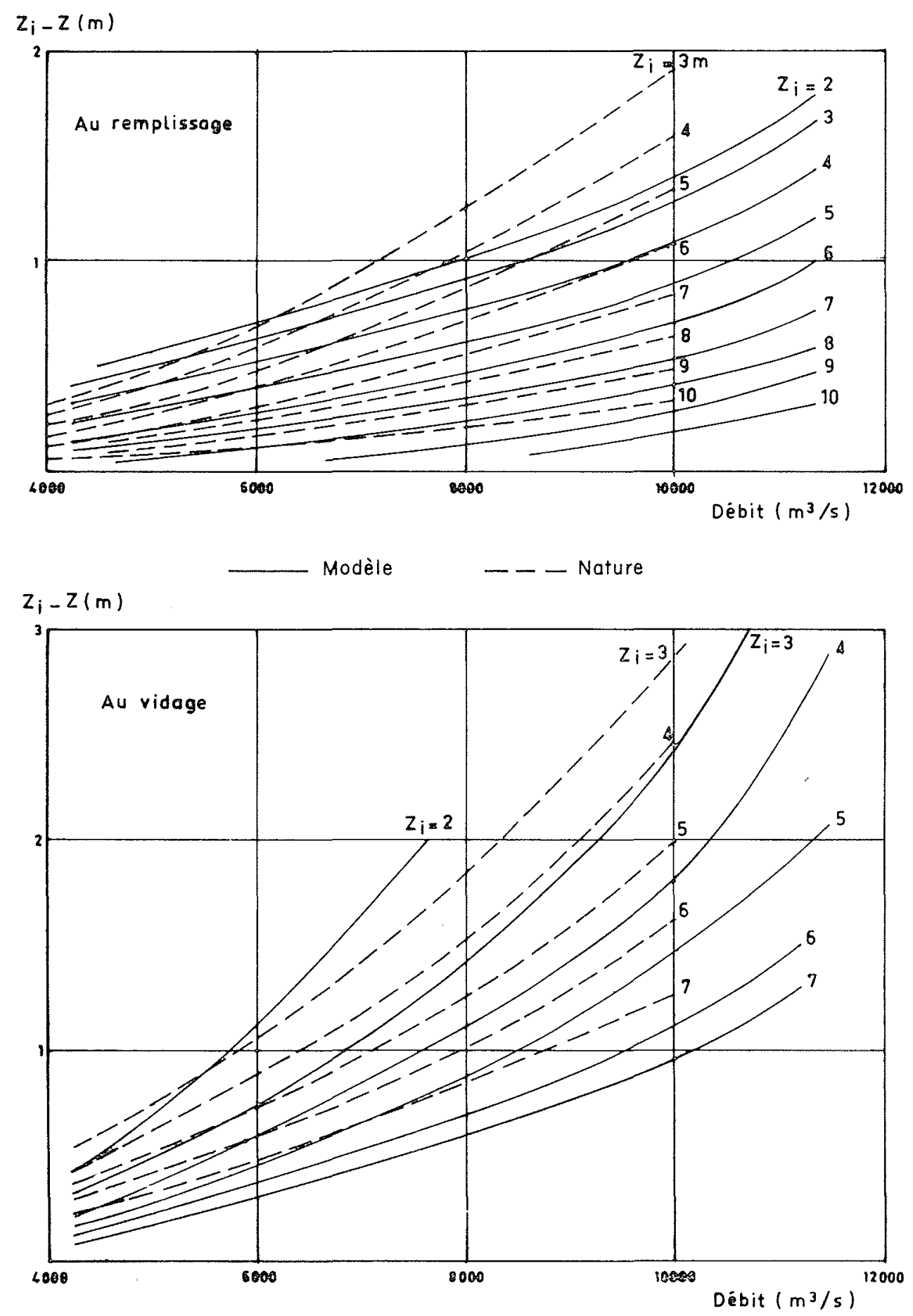

3/ Remous dans le bassin (— Modèle, _.... - utilisé par l'usine). 
- le remous et l'alimentation de l'usine;

- le profilage des ouvrages pour l'amélioration de l'écoulement à proximité de l'usine;

- les intumescences dues aux disjonctions et aux variations de fonctionnement de l'usine;

- le cheminement des eaux usées en Rance.

C'est sur ces points que peuvent être jugées les études sur modèle réduit. Malheureusement, les résultats prévisionnels du modèle ne peuvent vraiment être confrontés avec les observations nature que dans très peu de cas.

\section{La navigation en Rance}

Les cartes de courants, relevés sur le modèle de l'estuaire clans l'état naturel et avec l'usine en fonctionnement, ont permis de prévoir les heures de marée pour lesquelles les cycles de production peuvent modifier l'intensité des courants. L'étude a mis en évidence la nécessité de baliser certaines zones, dites «périlleuses», dans lesquelles la navigation est perturbée, en particulier lors des phases de vannage à proximité de l'écluse et dans la zone entre Bizeux et Cancaval.

Les relevés de courant in situ, entrepris depuis la mise en service de l'usine à la demande des Ponts et Chaussées de l'arrondissement de Saint-Malo au voisinage de l'écluse, peuvent constituer des éléments de la comparaison avec les résultats du modèle; malheureusement, ils correspondent à des conditions de fonctionnement très différentes de celles étudiées sur modèle.

Néanmoins, une comparaison peut être tentée entre:

- d'une part, les champs de courant relevés sur le modèle dans le cas d'un cycle en double effet, avec pompage en vive-eau de coefficient 95 ;

- d'autre part, les relevés én nature, entre les 10 et

13 octobre 1969 , en deux points, pour des cycles en double effet, avec et sans pompage en vive-eau.

Si les courbes de marée sont assez proches (fig. 1), les débits sont différents en phase: le vannage modèle se produit à pleine mer, tandis que le vannage nature a lieu environ une heure après. La comparaison (fig. 2), pour $4 \mathrm{~h}$ et $10 \mathrm{~h}$ après basse mer, montre cependant un accord satisfaisant sur les ordres de grandeur.

\section{Le remous dans l'estuaire}

Il est essentiel, pour une exploitation de l'usine rationnelle et optimale, de connaître à chaque instant le volume disponible dans le bassin et la charge de part et d'autre du barrage. A cet effet, le modèle a mis en relief un résultat intéressant: il existe un point du bassin où la connaissance de la cote suffit pour calculer le volume de l'estuaire, avec une erreur négligeable de l'ordre de $1 \%$ pour les deux sens de l'écoulement, dans l'hypothèse du vidage ou remplissage par tranche horizontale. Il s'agit de la tour marégraphique de la Grainjolais (Saint-Suliac) qui, depuis la mise en service de l'aménagement, définit le paramètre de pilotage de l'usine.

Le remous de l'usine dans l'estuaire, étudié sur le modèle systématiquement en fonction des débits, des niveaux et du sens de l'écoulement, est la différence entre la cote $Z_{i}$ correspondant à un vidage ou remplissage par «tranches horizontales», et la cote $\mathrm{Z}$ mesurée à $150 \mathrm{~m}$ de l'usine lors d'un vidage ou remplissage réel.

Le remous est actuellement défini par l'écart de niveau entre le plan d'eau à Saint-Suliac, qui représente le niveau $Z_{i}$ de vidage ou de remplissage par tranches horizontales, et le niveau réel $Z$ au point zéro «bassin ». C'est le remous étudié sur le modèle, à la seule différence que le niveau réel $Z$ n'est pas mesuré à $150 \mathrm{~m}$ de l'usine. En fait, ce point est fortement influencé par les écoulements locaux, de sorte que des mesures directes du remous à l'usine n'ont pas de sens.

Dans ces conditions, il n'a pas été possible de comparer les remous modèle et nature; l'étude s'est bornée à comparer le remous modèle au remous utilisé par les exploitants de l'usine pour préparer les cycles de fonctionnement. Cette comparaison est, apparemment, une source de déconvenue: la formulation utilisée pour simuler le remous conduit à une surestimation importante de celui-ci (fig. 3). Il est vrai que cet écart n'est grand que lorsque des débits importants traversent l'usine; or, les débits maximaux réels ne dépassent pas $5000 \mathrm{~m}^{3} / \mathrm{s}$.

En résumé, les remous relevés sur le modèle ( 1 à $3 \mathrm{~m}$ ) exagèrent l'importance des phénomènes, car ils correspondent à des débits de 7500 à $10000 \mathrm{~m}^{3} / \mathrm{s}$, doubles de ceux réellement turbinés. Pour les débits normalement turbinés, les remous sont inférieurs à $50 \mathrm{~cm}$; il semble qu'ils soient

\begin{tabular}{|c|c|c|c|c|c|c|}
\hline & & & & & & Tableau 1 \\
\hline \multirow{2}{*}{ DATE } & \multicolumn{3}{|c|}{ Nature } & \multicolumn{3}{|c|}{ MODÈLE } \\
\hline & $\begin{array}{l}\text { Débit } \\
\left(\mathrm{m}^{3} / \mathrm{s}\right)\end{array}$ & $\begin{array}{l}\text { Cote } Z \\
\text { d'arrêt }\end{array}$ & $\begin{array}{l}\text { Points de mesure } \\
\text { dans le bassin }\end{array}$ & $\begin{array}{l}\text { Débit } \\
\left(\mathrm{m}^{3} / \mathrm{s}\right)\end{array}$ & $\begin{array}{l}\text { Cote } Z \\
\text { d'arrêt }\end{array}$ & $\begin{array}{l}\text { Points de mesure } \\
\text { dans le bassin }\end{array}$ \\
\hline $\begin{array}{c}24 \text { janvier } \\
1968\end{array}$ & $\begin{array}{l}5000 \\
\text { (turbine } \\
\text { directe } \\
\text { arrêté } \\
\text { en } 30 \mathrm{~s} \text { ) }\end{array}$ & 7,10 & $\begin{array}{l}\text { Ecluse } \\
\text { Le Richardais } \\
\text { Troctin } \\
\text { Saint-Suliac } \\
\text { Mordreuc } \\
\text { Le Chatelier }\end{array}$ & $\begin{array}{c}5000 \\
\text { (turbine } \\
\text { directe) }\end{array}$ & 8,00 & $\begin{array}{l}\text { La Cage aux Moines } \\
\text { La Passagère } \\
\text { La Grainjolais } \\
\text { Mordreuc } \\
\text { Le Chatelier }\end{array}$ \\
\hline
\end{tabular}




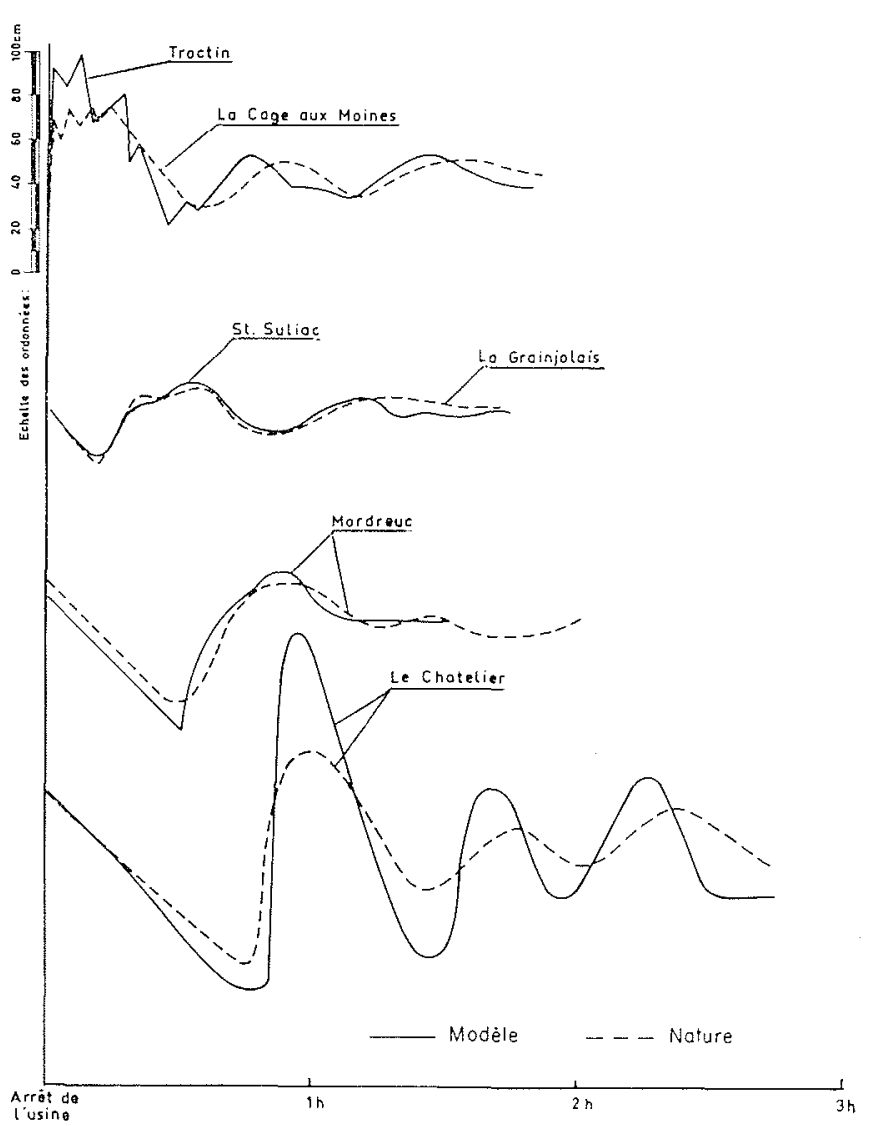

4/ Intumescences dues à un arrêt rapide de l'usine, en turbine directe à $5000 \mathrm{~m}^{3} / \mathrm{s}$, en $30 \mathrm{~s}$ (— Naturc ..... Modèle).

correctement pris en compte dans la préparation du programme de fonctionnement de l'usine puisque, en fin de cycle, le niveau de l'eau prévu est effectivement obtenu. Ceci prouve aussi que le niveau de Saint-Suliac est bien caractéristique de l'estuaire, puisque l'usine est pilotée à partir de ce seul niveau.

\section{Les intumescences}

Les variations brutales de débit, dues occasionnellement aux disjonctions et généralement aux variations de fonctionnement de l'usine, induisent des intumescences se propageant sur toute la longueur de l'estuaire.

L'étude sur modèle a permis de définir les consignes de sécurité en usage actuellement dans l'estuaire et les conditions optimales de remise en route des groupes travaillant en déchargeur. Pour estimer la valeur des résultats fournis par le modèle, des mesures in situ furent effectuées au cours d'un arrêt rapide des groupes le 24 janvier 1968. Les conditions de mesures nature sont consignées dans le tableau 1, ainsi que celles du modèle s'en rapprochant le plus.

La confrontation des relevés nature et modèle est particulièrement remarquable, comme le montre la figure 4. L'exagération de l'amplitude de l'onde au Chatelier, mesurée contre la porte de l'écluse, est due à la composition avec l'onde réfléchie, éliminée sur le modèle.

\section{Conclusion}

Ces quelques comparaisons laissent présager que les prévisions du modèle se produisent effectivement en nature, comme en témoignent les relevés d'intumescences effectués en modèle et nature dans des situations identiques.

Il est regrettable de ne pouvoir comparer les champs de courant nature et modèle; mais il est vrai que les conditions de fonctionnement de l'usine modèle sont très schématiques et que les réaliser simultanément avec une campagne de mesure n'apparaît pas comme une nécessité souhaitable par l'exploitant: le système usine-estuaire fonctionne correctement en appliquant les consignes déduites des essais sur modèle.

Il est, en revanche, très regrettable que le remous de l'usine ne soit pas mesuré avec précision. Ce paramètre, le $Z_{i}-Z$, fut un des critères de définition de l'usine, du fait de son importance sur le rendement de l'installation. Il parât donc aberrant, pour ceux qui l'ont étudié, qu'il soit devenu une quantité négligeable sans justification.

\section{Discussion}

Président: M. X. Ract-Madoux

M. le Président remercie M. Bonnefille et ouvre la discussion.

Un vif débat s'instaure entre $M$. Bonneficle et $M$. Marolleal sur la formule de calcul du remous $Z_{i}-Z$, introduite dans la détermination de la consigne d'optimisation de la production de l'usine, sur l'emplacement et le calage corrects du marégraphe de Saint-Malo, etc.

En définitive, conclut $M$. le Président, pour les débits d'équipement de l'usine, l'écart entre remous calculé et remous observé paraît assez modeste en pratique.
Il s'agit d'une dizaine de centimètres au maximum, répond M. BONNEFrlate, qui regrette l'emploi d'une formule «introduite par n'importe qui $\gg$.

Le problème du $\ll Z_{i}-Z$ 》 dit $M$. LE MENESTREL, avait fait, it l'époque, l'objet de discussions car les résultats trouvés sur les modèles de SOGREAH et «d'E.D.F.-SAINT-MALO» étaient nettement différents.

Par ailleurs, la formule représentative de la surface $G\left(Z_{i}-Z\right.$, $Q, h$ ou $Z$ ) introduite aux stades des études de la Rance dans les calculs de production optimale n'était pas celle de «n'importe qui ", 
mais déterminée empiriquement d'après les résultats des essais sur modèle. M. Le Menestrel pense que c'est la même formule qui est introduite dans les calculs actuels pour l'exploitation prévisionnelle de l'usine.

M. Gibrat intervient ensuite en ces termes:

J'insiste sur ce problème, bien qu'il soit sans importance sur la Rance, comme nous le savons. Par contre, il serait très important pour des installations beaucoup plus grandes. Or, nous ne sommes pas armés pour l'étudier à fond. Si un jour on remettait à l'étude le grand projet étudié il y a une dizaine d'années, un des premiers travaux à faire serait d'essayer de mieux comprendre ce problème.

Il faut d'ailleurs le distinguer d'un autre type de remous, qui est l'influence sur la marée même du fonctionnement des groupes. Souvent, on applique le mot «remous» aux deux phénomènes. Par ailleurs, on sait que l'infuence du barrage sur la marée en ce qui concerne la Rance est nulle. Donc, le mot «remous » n'est appliqué qu'à la première théorie, alors qu'il faudrait distinguer entre les deux phénomènes pour être sûr de ne pas se tromper dans le cas d'un grand ouvrage.

De son côté, M. BoNNEFILle souhaite qu'en raison de leur intérêt général les mesures du remous - défini comme il l'a indiqué dans son mémoire - soient reprises.

M. Bonnefille, observe M. le Président, s'était déjà fait connaître en 1956 , lors des Journées de l'Hydraulique consacrées aux «énergies de la mer», par l'importance de sa contribution aux études sur modèle de la marée et depuis cette date, la Revue La Houille Blanche lui a d'ailleurs réservé bien des pages.

Il nous montre aujourd'hui, non seulement qu'il n'a rien perdu de sa connaissance du problème, mais surtout qu'il a le souci, tout à fait louable, de confronter le résultat de ses recherches avec la réalité des phénomènes observés. C'est ce point qui me paraît essentiel et que je voulais souligner.

M. le Président clôt la discussion en remerciant les orateurs et les personnes qui l'ont animé et conclut:

Je voudrais, faisant allusion à ce qu'a dit le Président de séance de ce matin, M. WYarT, lorsqu'il a rendu hommage à tous ceux qui ont réalisé l'usine de la Rance: ingénieurs-conseils, constructeurs, entrepreneurs — sans oublier l'Equipement d'E.D.F. - je voudrais de mon côté déclarer que si nous constatons, aujourd'hui, qu'après six ans de service cet ouvrage fonctionne très bien, nous le devons, bien sûr, à nos collegues de la Production et du Transport. Mais je voudrais aussi rapprocher ces six années, des durées de vie qu'on adopte généralement dans les calculs économiques de l'hydraulique et qui sont soixante-quinze ans pour le Génic Civil, cinquante ans pour le matériel mécanique et trente ans pour le matériel électrique, de façon à conclure qu'un long avenir s'offre encore aux responsables de l'exploitation pour parvenir, comme l'a dit très justement M. LEFRANCOIS, non seulement à conserver l'intégrité de l'ouvrage, mais également à lui faire rendre toujours le maximum.

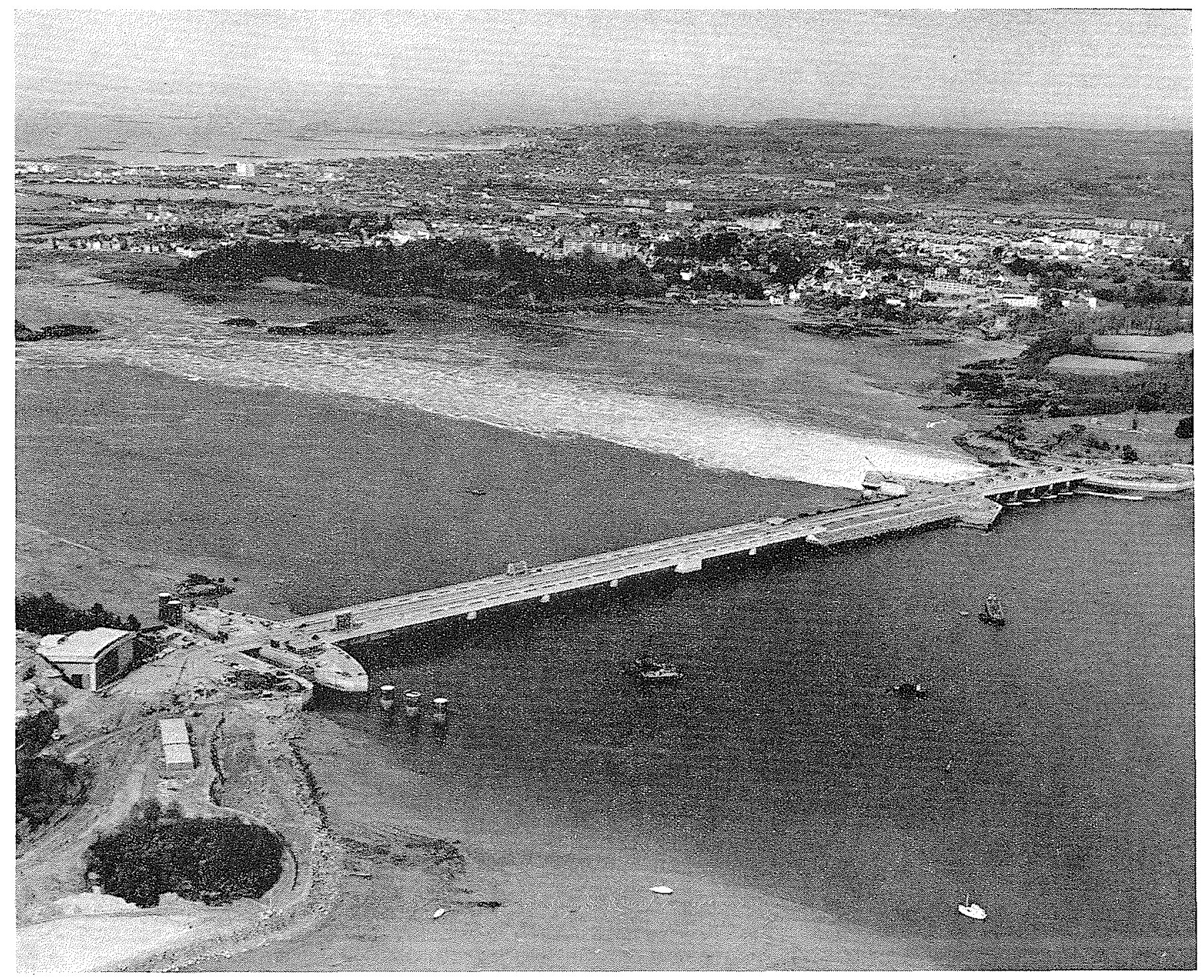




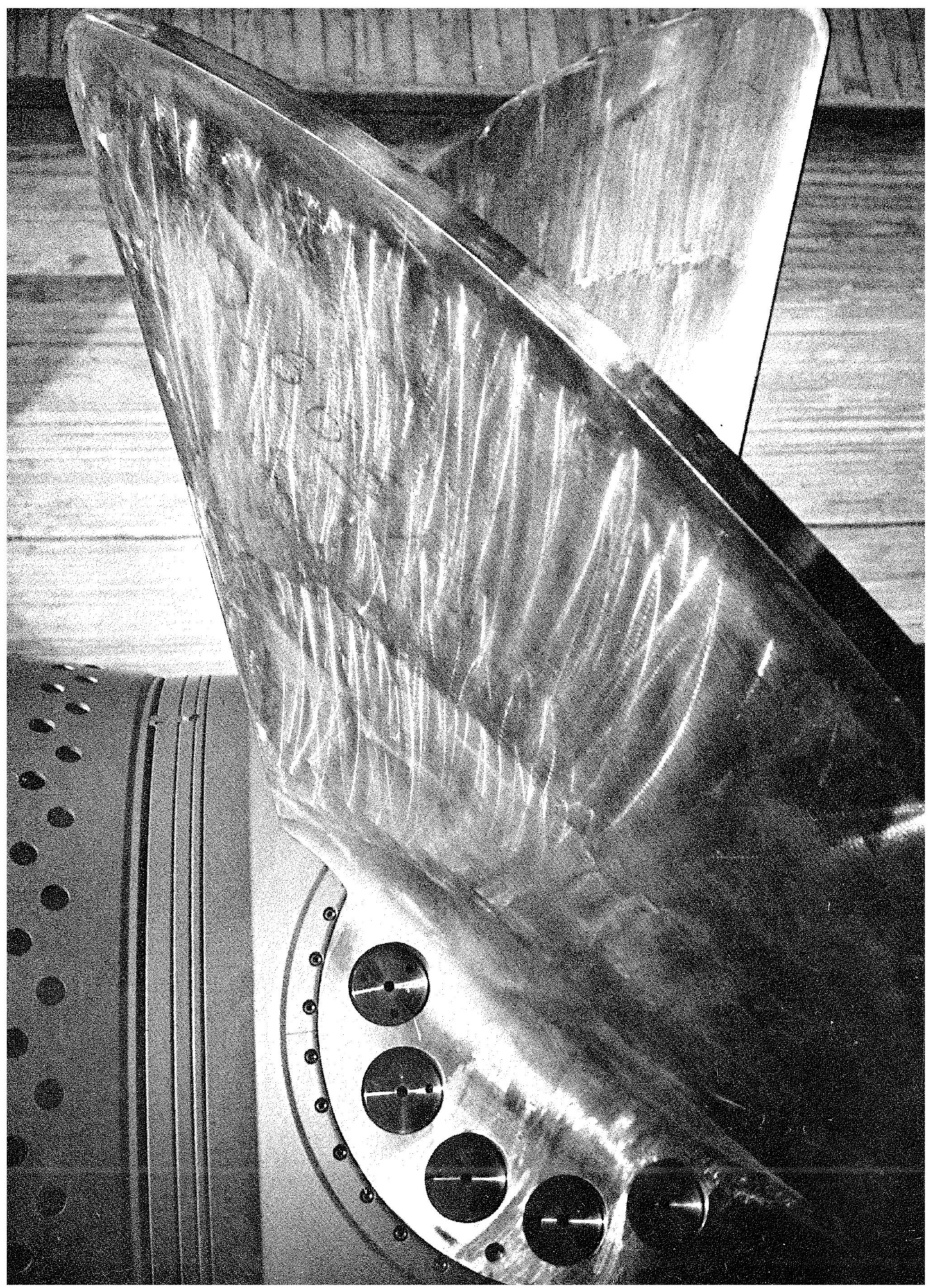

